\section{Check Digits for Detecting Recording Errors in Horticultural Research: Theory and Examples}

\author{
W.R. Okie \\ U.S. Department of Agriculture, Agricultural Research Service, Southeastern \\ Fruit and Tree Nut Research Laboratory, 21 Dunbar Road, Byron, GA 31008
}

\section{E.G. Okie}

Department of Information Technology, Radford University, Radford, VA 24142

Additional index words. breeding, error detection, modular arithmetic, data collection, modulus

\begin{abstract}
Check digit technology is frequently used in commercial applications such as shipping labels and credit cards to flag errors in numbers as they are used. Most systems use modular arithmetic to calculate a check digit from the digits in the identification number. Check digits are little used in horticultural research because the guidelines for implementing them are neither well known nor readily accessible. The USDA-ARS stone fruit breeding program at Byron, Ga., plants thousands of trees annually, which are identified using a 2-digit year prefix followed by a sequential number that identifies the tree location in the rows. Various records are taken over the life of the tree including bloom and fruit characteristics. Selected trees are propagated and tested further. To improve the accuracy of our records we have implemented a system which uses a check number which is calculated from the identification number and then converted to a letter that is added onto the end of the identification number. The check letter is calculated by summing the products of each of the digits in the number multiplied by sequential integers, dividing this sum by 23, and converting the remainder into a letter. Adding a single letter suffix is a small change and does not add much complexity to existing data collection. The types of errors caught by this system are discussed, along with those caught by other common check digit systems. Check digit terminology and theory are also covered.
\end{abstract}

Horticultural and agronomic research often involves record keeping and data collection for large numbers of experimental subjects. For example, many breeding programs grow and evaluate thousands of individual plants annually. Research with perennial crops such as fruit trees may involve repeated measures on certain plants over time. Frequently, record collection is done by a person who enters the identification number of the experimental subject at the time of data collection. Any time numerical values are entered by hand, the potential for error exists, particularly where longer numbers are used. Such errors include misreading labels, mis-remembering numbers prior to recording them, and mis-entering the number if a keypad is used. If the numbers are written down, there is potential error when they are read or transcribed later.

Check digit technology involves the use of numbers or characters calculated from the identification number and appended to it, in order to detect errors that occur as the number is used. With the development of computer technology, such check digits are commonly used in commercial settings, usually invisibly to the end-user. Shipping labels, routing numbers on checks, and driver's license and credit card numbers all use check digit technology. This technology could be more widely used in horticultural research if the theory behind it

Received for publication 4 May 2005. Accepted for publication 11 July 2005. the remainder. The following examples show $\bmod$ in use: $11 \bmod 5=1$

$$
-11 \bmod 5=4 \text {. }
$$

In general, the right operand of a mod operator (i.e., each 5 above) is called the modulus, but unfortunately there is no agreed upon name for mod's left operand. In the second example the quotient is -3 , which results in a remainder of 4 . Since adding a multiple of the modulus to the left operand of mod leaves the result unchanged, this equation can be checked by verifying that $(-11+15) \bmod 5=4$. See Knuth (1997) for more details on modular arithmetic.

Although the term check digit implies that the symbols used for error checking are decimal digits, in many systems other characters, such as letters and symbols, are used for this purpose. For simplicity, however, we use the term check digit regardless of the characters used. Similarly in some cases we use the term check digit even if the system in fact has several check digits. Also for simplicity, at some times we use the term identification number to refer to a number that does not contain a check digit and at other times to refer to a number that does contain one. This is only done when the precise meaning is either unimportant or clear from context. Sometimes we use the term word to refer to a sequence of characters such as an identification number.

Three general categories of errors are typically studied in relation to check digit usage:

Transcription is replacing one digit by a different value, as in changing 111 into 121 .

Transposition is swapping the values at two locations, as in changing 1234 into 4231.

Phonetic is replacing a number with a similar sounding number, as in changing 15 to 50 .

Variants of these errors differ in how many digits are affected and their relative positions.

Transcription variants. Single digit is replacing a single digit, as in changing 111 into 121 ; twin is replacing with the same value each of two identical adjacent digits, as in changing 1111 into 1221; multiple identical transcriptions at any location is changing 122112221212 to 133113331313 ; jump twin is replacing with the same value each of two identical digits separated by one digit, as in changing 131 to 232 .

Transposition variations. Adjacent transposition is swapping adjacent values, as in changing 12 into 21; jump transposition is swapping values that are separated by one digit, as in changing 1213 into 1312; transposition at any location is like changing 0123456 to 0523416 .

In empirical studies of actual error occurrences reported by Verhoeff(1969) andBeckley (1967), most errors were single transcription errors, and these errors combined with adjacent and jump transposition errors comprised $>90 \%$ of all errors found in the studies. These are the primary errors considered in the creation and analysis of check digit systems. Although these are important classes of errors, different data may have characteristics that make other errors more important. 
The error-checking capability of a system is determined by the interaction of a number of factors, including the number of check digits, the range of possible values of each of the digits in the number and the check digits, and the values of the weights and the modulus $P$. Also relevant in some cases is the location of the check digit, which is usually located on the right of the number. In cases in which an existing system of identification numbers is being extended by adding check digits, most of these factors are predetermined, and the only choices to be made are the values of the modulus and the weights. As explained below, a system will in general detect the most errors if these values are chosen so that the weights are unique and so that $P$ shares no divisors (including itself) with any of the weights or with any of the possible digits of the identification number. One common way to meet these conditions is to make $P$ both prime and larger than all of the digits and weights. So, for example, for identification numbers that have 10 or fewer decimal digits, these conditions can be met by choosing $P$ to be 11 and the weights to be the integers in the range from 1 up to the length of the number. This is essentially the system found in the ISBN system which publishers use to identify books. Of course, when $P$ is $>10$, the remainder that occurs can be $>9$, and as a result decimal digits must be supplemented with symbols.

\section{ARS Byron Implementation}

The USDA-ARS stone fruit breeding program at Byron, Ga., typically plants 5000 seedling trees annually. Trees are identified using a 2-digit year prefix followed by a sequential number that identifies the tree location in the rows. Various records are taken over the life of the tree including bloom and fruit characteristics. Selected trees are propagated and tested further. Throughout this multiyear process, record-keeping errors creep in. Most common are transposition errors, such as recording 99-1234 as $99-1243$ or $99-1324$. To improve the accuracy of our records we have implemented a system that uses a check number that is calculated from the identification number and then converted to a letter which is added onto the end of the identification number. Adding a single letter suffix is a small change and does not add much complexity to existing data collection.

The check letter is calculated by summing the products of each of the digits in the number by weights that are sequential integers, dividing this sum by a modulus of 23 , and converting the remainder into a letter. Because the modulus is 23 , the 23 possible remainders range from 0 to 22. Remainders are matched sequentially with the sequence of letters a to $z$, except that $i, 1$, and o are not included because they are likely to be misread. In the example above the sum and check letter for 99-1234 would be calculated as follows: $(1 \times 9)+(2 \times 9)+(3 \times 1)+(4 \times 2)+$ $(5 \times 3)+(6 \times 4)=77$, and $77 / 23=3$ remainder 8 (i.e., $77 \bmod 23=8$ ), which maps to check digit $\mathrm{j}$. Thus the new identification number would be $99-1234 \mathrm{j}$. As noted in the previous section, we refer to the added character as a check digit, even though it is actually a letter and not a decimal digit.

Once a check digit has been added, an identification number can be checked by recalculating what the check digit should be and comparing that with what is found. One reason for selecting sequential integers as weights is the ease of remembering them and, if necessary, recalculating the check digit in the field. A mismatch, of course, indicates that an error has occurred. Errors would be revealed, for example, in the numbers $89-1234 \mathrm{j}$ and 99 $2134 \mathrm{j}$ because the check digit of each should be the letter $h$.

Although a mismatch between the check digit and the rest of the identification number definitely indicates that an error has occurred, a match does not definitely indicate that no errors have occurred. Some errors, such as recording 99-1234 $\mathrm{j}$ as $89-1234 \mathrm{~h}$, result in an identification number with a matching check digit. Thus, a match indicates either that no error occurred or that an undetected error occurred. Although there is no way to tell whether a number whose check digit matches is indeed correct, the assumption that it is correct is a safe one because we begin with the assumption that correct data is more likely than errors.

The design of this check digit system began with identification numbers made up of six decimal digits, and required a choice of the set of characters for the check digits and the values of the modulus and weights (i.e., the values of the divisor and the multipliers for the digits). Values were chosen for these interacting characteristics to create a system that detects a broad set of errors while being simple and reliable in use.

To keep the system simple, the set of check digit characters was chosen to be a set of lower case letters. Using both upper and lower case letters as well as decimal digits would have provided a larger set of check digits which would cause fewer numbers to share the same check digit. However it would also have resulted in more potential errors since some characters and numbers are easily confused. Decimal digits were avoided to prevent confusion with the tree number itself, particularly to avoid confusion in legacy records with no check digit. As explained later, prime divisors give better error detection and so the divisor 23 was chosen as the largest prime $<26$, the number of lower case letters. The multipliers for the digits were chosen to be unique and all less than the divisor.

Based on the conditions given in the next to last section, this set of choices provides a system that will detect, among others, all transcription errors in a single digit as well as all transposition errors (i.e., those involving swapping two values) at all positions except the first and last. Briefly, for both categories the error is detected because one or two weights are multiplied by a different value which results in a different sum. Because of the choices of weights and modulus, the change in the sum cannot be a multiple of the modulus, and so the mod operation will result in a different remainder. This is true even when the error involves the check digit itself.
Consideration was given to using more than one check digit which would have provided the ability to correct certain errors, but this would have required a more significant change to the underlying numbers since it would increase the size by one third rather than by one sixth. It would also have introduced potential for errors of transposing check letters.

When it is necessary to calculate our check digit by hand, we can simplify the calculation so that it uses only ten additions rather than six multiplications and five additions (Hamming, 1980). Table 1 illustrates the method, which works for systems using sequential weights starting at 1 , with an example using first regular arithmetic and then using even simpler modular arithmetic. The first column of the table identifies the row number; the second column contains the digits of the identification number, which must be listed in reverse order. The third and fourth columns show the simplified calculation using regular arithmetic. The desired result of this calculation is 8 , which is $(77 \bmod 23)$, and the 77 is the value found in row 6 of column four. The key to this procedure is that in column four, the value in each row is repeatedly added to each of the values in the rows below, and so, for example, the value of 4 in row one is included 6 times (i.e., $6 \times 4$; note weight is 6 also) in calculating the value 77 . In columns 5 and 6 the calculation in columns three and four is repeated, but modular arithmetic is used for partial calculations throughout rather than just once for the final result. Thus, for example, in columns 3 and 4 the values 20 and 10 in rows three and four are added to give 30 in row four, while in columns 5 and 6 the values 20 and 10 result in a value of 7 in row four of column 6 when modular 23 arithmetic is used (i.e., 30 $\bmod 23=7$ ).

\section{Designing a System}

When developing a check digit system, the range of values that will be allowed for each data digit must be decided. Many systems are based on either binary (digits have the value either 0 or 1 ) or decimal numbers (digits have values ranging from 0 to 9 ). Others, like the breeding program system described above, do allow more than 10 values at some or all positions by using another set of symbols, either in place of or in addition to the decimal numerals. Using more symbols at each position of course makes it possible to represent more information with fewer digits. Not surprisingly the most common additional symbols are letters, upper case, lower case, or both. Sometimes characters other than numerals and letters are used. Binary numbers are used heavily in digital electronic systems, but when the data are to be directly viewed by humans without intervening interpretation, a base of 10 or larger is typically used.

There is no restriction that all digits use the same range of values or that different digits use the same representation. In our breeding program system, for example, the data digits are all base 10 with all values being represented by decimal digits while the check digit is of 
base 23 with all values being represented by letters. The Code 39 system discussed below uses 10 digits, 26 letters, and 3 other characters as a set of symbols for base 39 numbers. An additional version of the system allows an additional 4 symbols in the check digit which makes it a base 43 number while all of the other digits are base 39 numbers. In addition, some systems are designed to have alphabetic information, such as a person's initials, in the identification number. In some systems in which the modulus is larger than 10 , no additional symbols are used. Instead, an identification number whose check digit would be 10 or greater is simply not allowed to be used. Of course this technique would not be feasible when adding check digits to an existing system, but it could be used when creating a new system. Various encodings of numbers to symbols are commonly used for barcodes in different applications and countries.

Another choice in designing a system is the size (designated as $n$ ) of the identification number, including any check digits. In general, for a fixed number of check digits, smaller values for $n$ allow for better error detection because the redundant check digit information is a larger proportion of the entire number and the size of the set of values that share the same check digit is smaller. In some systems there is an upper limit on the size of the word because, for example, each position must have a unique weight that is less than the modulus. In these systems the choice of the modulus determines the maximum length of the word. In other systems, because the weights do not have to be unique they can be repeated, and the word can be of any length.

In the example systems described here, the check digits have all been at the end of the number. However, there is no requirement for this, and in some cases it may be preferable to have them elsewhere. For example, if it is desired to sort the identification numbers into groups that have the same check digit, then it might be more useful to put the check digit first. In other cases, there might be natural groups within the data and it could be useful to place the check digit between a pair of these groups.

\section{Examples of Commercial Application}

It is helpful to review check digit systems developed for commercial use and to consider the percentage of specific errors that each can detect. In a modulus 10 system with decimal digits, for example, a digit position that has a weight of 2 will detect all transcription errors at that position, except those with a size of 5 . Now, since there are 10 possible digits that can occur in that position, and since for each of these 10 digits there are 9 possible errors, we can conclude that a total of 90 different errors can occur at that position. Of these 90 , exactly 10 cannot be detected because for each of the 10 possible digits that could be at that position, there is exactly one error that has a size of 5. Thus, 80 out of 90 , or $88.9 \%$, of the possible errors at that position can be detected. Of course this analysis does not take into account the actual likelihood of an error; it simply calculates what percentage of possible errors can be detected. Table 2 shows the percentage of errors detected for some of the systems described below.

A convenient way of describing a check digit system is to list the weights of all of the digits of the identification number, and to include in this list a weight for the check digit itself. When this is done, the weight of the check digit is assigned so that the mod of the sum of all of the weight and digit pairs (including the check digit pair) will be zero. Following this method, the weights for our breeding program system, for example, would be listed as (1, 2, $3,4,5,6,-1)$, where -1 is the weight of the check digit. For more detailed information on error detection see the next section.

The popular Code 39 system repeats a single weight. The weight of the check digit is -1 , the other digits all have a weights of 1 , and the modulus is 43 (Gallian, 1996). Thus, this system effectively uses the mod of the sum of all of the digits as the value of the check digit. Since $P$ is larger than all possible digit values, it can detect all single digit errors. However, transposition errors that do not involve the check digit are not detected since they can have no effect on the checksum. On the other hand, transposition errors involving the check digit are detected by the system.

Several UPC codes are used to identify retail goods. One of these is a 12 digit, modulus 10 scheme whose repeating weights are $(3,1,3$, $1,3,1,3,1,3,1,3,1)$, and which uses decimal digits. Since 10 is larger than all digits and since 3 and 1 are relatively prime to 10 , this system detects all single digit errors. It detects adjacent transpositions unless the size of the error is 5 . Such an error is not detected because the difference between adjacent weights is 2 and the product of this difference and the error size gives 10. Jump transitions are not detected because the difference between weights separated by one position is 0 (Gallian, 1996).

In systems with three repeating weights, the weights are normally chosen to be relatively prime to $P$ allowing these systems to detect all single-digit errors. The weights are also

Table 1. Shortcut method for calculating weighted sums for breeding program example for tree number 99-1234. Results calculated via columns 5 and 6 are comparable to those of column 3 and 4 but involve simpler arithmetic. Note that the results at the bottom of columns 4 and 6 are comparable since 77 mod $23=8$.

\begin{tabular}{|c|c|c|c|c|c|}
\hline \multirow[b]{2}{*}{ Row } & \multirow{2}{*}{$\begin{array}{c}\text { Digits } \\
\text { of number } \\
\text { in reverse }\end{array}$} & \multicolumn{2}{|c|}{ Regular arithmetic } & \multicolumn{2}{|c|}{ Mod 23 Arithmetic } \\
\hline & & $\begin{array}{l}\text { Running sum } \\
\text { of column } 2\end{array}$ & $\begin{array}{l}\text { Running sum } \\
\text { of column } 3\end{array}$ & $\begin{array}{l}\text { Running sum } \\
\text { of column } 2\end{array}$ & $\begin{array}{l}\text { Running sum } \\
\text { of column } 5\end{array}$ \\
\hline$\overline{1}$ & 4 & 4 & 4 & 4 & 4 \\
\hline 2 & 3 & 7 & 11 & 7 & 11 \\
\hline 3 & 2 & 9 & 20 & 9 & 20 \\
\hline 4 & 1 & 10 & 30 & 10 & 7 \\
\hline 5 & 9 & 19 & 49 & 19 & 3 \\
\hline 6 & 9 & 28 & 77 & 5 & 8 \\
\hline
\end{tabular}

Table 2. Percentage of errors of various types detected by common check digit systems. $P$ represents the modulus. In several of the systems the results depend on the length of the identification number. The length is assumed to be 9 for the bank system, and 10 for the passport, Code 39 and 4 -weight systems. Eight phonetic errors are considered, one for each of the digits from 2 to 9 .

\begin{tabular}{|c|c|c|c|c|c|c|c|}
\hline \multirow[b]{3}{*}{ System (weights) } & \multirow[b]{3}{*}{$P$} & \multicolumn{6}{|c|}{ Error type $(\%)$} \\
\hline & & \multicolumn{3}{|c|}{ Transcription } & \multicolumn{2}{|c|}{ Transposition } & \multirow[t]{2}{*}{ Phonetic } \\
\hline & & Single & Twin & Jump twin & Adjacent & Jump & \\
\hline UPC-12 $(3,1, \ldots)$ & 10 & 100 & 88.9 & 88.9 & 88.9 & 0 & 100 \\
\hline Passport $(7,3,1, \ldots, 9)$ & 10 & 100 & 49.4 & 66.7 & 88.9 & 88.9 & 100 \\
\hline Banking $(3,7,1, \ldots)$ & 10 & 100 & 55.6 & 63.4 & 88.9 & 88.9 & 100 \\
\hline 4-weight $(1,3,9,7, \ldots)$ & 10 & 100 & 88.9 & 0 & 88.9 & 88.9 & 100 \\
\hline 4-weight $(1,3,7,9, \ldots)$ & 10 & 100 & 49.4 & 88.9 & 88.9 & 88.9 & 100 \\
\hline Credit card/IBM & 10 & 100 & 93.3 & 87.7 & 97.8 & 0 & 87.5 \\
\hline P.T.T. & 10 & 100 & 95.6 & 95.6 & 96.3 & 96.3 & 95.8 \\
\hline ISBN $(1,2, \ldots, 9,10)$ & 11 & 100 & 88.9 & 100 & 100 & 100 & 88.8 \\
\hline Powers of $2(1,2,4, \ldots)$ & 11 & 100 & 100 & 100 & 100 & 100 & 100 \\
\hline Byron breeding program $(1,2,3,4,5,6,-1)$ & 23 & 100 & 100 & 100 & 100 & 100 & 100 \\
\hline Code $39(1,1,1, \ldots,-1)$ & 43 & 100 & 88.9 & 87.5 & 11.1 & 12.5 & 100 \\
\hline Dihedral & --- & 100 & 100 & 94.2 & 100 & 94.2 & 100 \\
\hline
\end{tabular}


typically chosen so that the difference between weights separated by one position is not 0 , allowing the detection of most transposition errors. American bank routing numbers (leftmost 9 digits on a check) use a 9 digit modulus 10 decimal system with weights $(3,7,1,3,7$, $1,3,7,1)$, or equivalent weights $(7,3,9,7$, $3,9,7,3,9)$. Passport numbers in the United States and some other countries use a ten digit modulus 10 system with weights $(7,3,1,7,3,1$, $7,3,1,9)$ in which the check digit is appended to the 9 digit passport number on a machinereadable line. In both of these systems, since the difference between weights separated by one position is 2,4 , or 6 , the systems detect all jump transition errors except those whose size is 5. Similar performance will result from any set of weights that are unique and relatively prime to $P$.

Systems with four repeating weights all relatively prime to the modulus of 10 (such as 1 , $3,7,9$ and $1,3,9,7$ ) also detect all single digit errors and some of the transposition errors. With the length 3 and length 4 systems, the length of the word and the order of the weights affect the proportion of some types of errors that can be detected since they determine the proportion of combinations of weights that will have a sum equal to 10 . The second 4-weight system given above, for example, detects no jump twin errors; however, the first detects many, but not all, of these errors (Gallian 1996).

Asystem that does not use repeating weights is the International Standard Book Numbering (ISBN) system used by publishers to identify books. This 10-digit system includes a single check digit, weights of 1 to 10 (or, equivalently, weights of 10 to 1 ), and a modulus of 11 , which is the smallest prime that is larger than all of the decimal digits. It uses the character $\mathrm{X}$ for a check digit value of 10 , but values of $\mathrm{X}$ are not allowed in the other positions of the number. The rightmost digit is the check digit and its value can easily be calculated as the weighted sum, modulus 11 , of the first nine digits because having a weight of 10 is equivalent to having a weight of -1 , since $-1 \bmod 11=10$. This system detects all single digit errors and all transposition errors (Gallian, 1996). Beginning in 2005 this system will be superceded by ISBN-13, which will use 13 digits, including a mod 10 check digit on the right, and repeating weights of 1 and 3 .

The weights (except perhaps for the weight of the check digit) in the ISBN and breeding program systems form an arithmetic series (i.e., at each position except the first, the weight at that position is formed by adding a constant to the weight at the previous position). Systems based on geometric series (which multiply by a constant to get the next weight) are also used. Airlines use a modulus 7 system with weights of decreasing powers of ten for the identification number and -1 for the check digit which is the rightmost digit. Since the value of the identification number itself (without the check digit) is identical to the weighted sum of the digits, the check digit is simply the remainder when the original number (without the rightmost digit) is divided by 7 . This system detects most but not all single digit and transposition errors. U.S.
Postal Service(USPS) money orders and VISA traveler's checks use modulus 9 systems with weights that are also powers of ten. Modulus 9 systems using as weights powers of $10 \mathrm{can}$ alternately be calculated by simply summing the digits mod 9. Because the modulus in these systems is less than 10 , they do not detect all single digit errors (Gallian 1996).

Tracking numbers for United Parcel Service (UPS) and USPS use Code 128, a barcode system, which uses a modulus of 103 for calculating a check digit. Standard commercial bar coding software calculates these values using sequential weights starting at 1 . Additional characters are not needed to represent the check digit in this system since it encodes the check digit in the barcode but it does not print the check digit as part the visible number.

A system used by some German banks uses powers of two as weights and a modulus of 11 and detects all of these errors: single transposition, adjacent and jump transposition, twin and jump twin, and phonetic (Gallian 1996). State driver's license numbers use a variety of systems which detect a varying range of single and transposition errors. Surprisingly, many states use systems that do not even detect all single digit errors (Gallian, 1996).

Some modulus systems use calculations that are more complex than simply multiplying by weights. One such system was developed by IBM and is used by credit card companies. For a number with an even number of digits such as a 16-digit card number, digits $d_{1}, d_{3}, \ldots, d_{15}$ are multiplied by $2 \bmod 9$ and then summed. The other digits $d, d, \ldots d$ are summed without weighting. The check digit $d_{16}$ is the total of the two sums mod 10. It detects all single digit errors but only most transposition errors. Another system, known as P.T.T., which uses modular arithmetic but which does not simply multiply by weights, is used by some German banks (Gallian, 1996).

A different class of error detection system, developed independently by Verhoeff and Gumm, does not use modular arithmetic at all. Although these systems, which use dihedral groups, are beyond the scope of this paper, they are of note, since one such system can detect all single digit errors and all adjacent transpositions in decimal identification numbers, all without introducing any additional symbols, which is not possible using modular arithmetic (Gallian, 1996; Gumm, 1969; Verhoeff, 1969). Kirtland (2001) gives a clear presentation of this method.

In contexts such as for use in digital equipment, binary systems, in which all digits are either 0 or 1 and the modulus is 2 , are common. Binary systems have a rich theory of error detection and correction, and the codes used in computer storage and satellite transmissions, for example, are quite sophisticated. Compact discs for music recordings, for example, use 64 check bits for each 192 data bits so that data can still be recovered despite flaws in the disc or errors that occur during the reading process. In many applications, when an error is detected it is possible to ask that the original data be retransmitted as is done on the Internet. However, in some applications re-transmission is not feasible, and it is necessary to have correction sufficient for a high-error environment. For the Mariner 9 spacecraft which photographed Mars, for example, 6 data bits were encoded with 26 check bits to correct up to 7 bits, in order to compensate for a very noisy transmission environment (Hill, 1989).

\section{Error Detection}

When analyzing particular errors and the conditions that a check digit system must satisfy to detect them, it is useful to have a generic representation for the system. Using $d, \ldots, d$ to represent, from left to right, the digits of the identification number (including the check digits) and $w_{1}, \ldots, w_{n}$ to represent the values of the corresponding weights, in a check digit system based on modular $P$ arithmetic, the value of the check digit is chosen so that the following equation holds:

$$
\left(\sum_{i=1}^{n} w_{i} \times d_{i}\right) \bmod P=0
$$

For clarity, note that an alternate representation of Eq. 1 is the following: $\left[\left(w_{1} \times d_{1}\right) \times\left(w_{2}\right.\right.$ $\left.\left.\times d_{2}\right)+\ldots+\left(w_{n} \times d_{n}\right)\right] \bmod P=0$

As an example, when the breeding program system is put in this form, $n$ has the value 7, the weights satisfy the equations $w_{i}=i$ for positions 1 to 6 and $w_{7}=-1$. In other words, the digit at position $i$ has a weight of $i$, and the weight of the check digit is $-1:\left[\left(1 \times d_{1}\right)+\left(2 \times d_{2}\right)+(3\right.$ $\left.\times d_{3}\right)+\left(4 \times d_{4}\right)+\left(5 \times d_{5}\right)+\left(6 \times d_{6}\right)+((-1) \times$ $\left.\left.d_{7}\right)\right] \bmod 23=0$.

To see that the weight of the check digit is correct, note that adding the check digit (that is, digit $d_{7}$ ) to both sides of Eq. 1 results in the check digit being equal to the sum, $\bmod 23$, of the six digit-weight products, which is the original formulation of the system: $d_{7}=[(1 \times$ $\left.d_{1}\right)+\left(2 \times d_{2}\right)+\left(3 \times d_{3}\right)+\left(4 \times d_{4}\right)+\left(5 \times d_{5}\right)+$ $\left.\left(6 \times d_{6}\right)\right] \bmod 23$

When values from an identification number are substituted into Eq. 1, the value of the expression on the left side of the equation is called the checksum of the number. A nonzero checksum, of course, indicates that an error has occurred, and, as we discuss in the last section, the value of this checksum can provide information about the nature of the error. When a number has a checksum of zero, then either the number is correct or an undetectable error has occurred.

In Eq. 1 any check digits in the identification number are not distinguished from the other digits, and so there is no indication how many check digits the number contains, in what position they are located, or how the check digits are calculated. Instead we simply assume that the values of any check digits are chosen to make the equation true and that their location is not relevant. Treating check digits as indistinguishable from the other digits does make analyzing the system much easier. In actual practice, of course, the positions of the check digits must be specified, along with formulas for calculating them.

Using Eq. 1 we can analyze the effects of different errors and find the conditions needed to detect them. In Table 3 , based on results in 
Gallian (1996) and Wild (1968), are conditions that a system must meet to detect each type of error. Column 3 gives general conditions under which the specified error will be detected, and columns 4 and 5 show simpler conditions that result when $P$ meets certain simplifying requirements. Note that throughout the table each condition guarantees that a particular error will be caught only if that error is the only error in the word. However, when several errors occur, there is no guarantee that all will be detected, even if the system meets the conditions required to detect each type of error individually.

In the breeding program system $P$ is 23 , which is prime and larger than all the digits, allowing us to use column 5 of Table 3 . Given our weights, it is easy to see that this code meets the conditions to detect all single transcription errors. It also detects all multiple identical transcription errors at any combination of locations except for the first and last position (unlikely in our case) since the weights of these two positions are 1 and -1 , respectively. The code also detects all transposition errors between two digits in any positions and all phonetic errors. Of course, it is only guaranteed to detect one such error. If several errors have occurred, then detection is not guaranteed.

To better understand the conditions in Table 3 , it is useful to focus on the errors that are not detected by a system. In a modulus $P$ system, an error is not detected if the error changes the checksum by a multiple of $P$. More specifically, if a single error occurs at a given position then that error is not detected if product of the weight at that position and the size of the error is a multiple of $P$, and the error is detected if this product is not a multiple of $P$. The size of an error (represented by $S$ below) at a given position is the value of the incorrect digit minus the value of the corresponding digit in the valid word. In some contexts where the sign of an error is not relevant we discuss the size as being positive when in fact it could be either positive or negative. A key goal in choosing $P$ and the weights, therefore, is to minimize the set of errors that change the checksum by a multiple of $P$, thereby causing the error to be undetected. Stated in reverse, the goal is to maximize the set of errors that cause a checksum change that is not a multiple of $P$.

As mentioned above, the condition for detecting a single transcription error in position $i$ is that the product of the weight and error size is not a multiple of $P$, which is represented in column 3 as $\left(w_{i} \times S\right) \bmod P \neq 0$. Since this condition involves the weights, error sizes, and $P$, all three of these factors must be considered together when analyzing the error correcting capability of a system. To simplify analysis, it is useful to divide this single condition into two, one involving $P$ and the weights and the other relating $P$ and the error sizes. The relation between $P$ and the error sizes is immediately obvious: $P$ must be different from all of the error sizes, for if it equaled any of them then the product of the weight and size would be a multiple of $P$ and the condition would not hold. Considering only positive $\mathrm{P}$ and assuming that error sizes range from 1 to the size of the largest digit, then $\mathrm{P}$ will be different from all of the error sizes when it is larger than all of the sizes, that is, when $P>S$. A simple way to guarantee that $P>S$ for all $S$ is to require $P$ to be larger than all possible digits at position $i$ since the largest error, which results when the smallest digit is replaced by the largest, can be no larger than the largest digit. This condition is represented in columns 4 and 5 as $P>d_{i}$.

When the condition $P>d_{i}$ holds, the condition $\left(w_{i} \times S\right) \bmod P \neq 0$ is guaranteed to hold if the greatest common divisor of $w_{i}$ and $P=1$, that is, if their only common factor is 1 (i.e., $w_{i}$ and $P$ are relatively prime). In column 4 of Table 3 , this condition is represented as $\operatorname{gcd}\left(w_{i}\right.$, $P)=1$. To see that this condition guarantees that the error can be detected, consider that if $\left(w_{i} \times S\right) \bmod P=0$, then $P$ must be a factor of the product $w_{i} \times S$, but when $P>S, P$ is not a factor of $S$ and so $w_{i}$ and $P$ must have a common factor. Thus, if $w_{i}$ and $P$ do not share a common factor, then $\left(w_{i} \times S\right) \bmod P \neq$ 0 . One further restriction of $P$, choosing $P$ to be a prime number, results in an even simpler relation between $P$ and the weights. When $P$ is prime, then $w_{i}$ and $P$ are guaranteed to be relatively prime unless $w_{i}$ is a multiple of $P$, that is, unless $w_{i} \bmod P=0$. Thus, as shown in column 5 of the table, the condition $w_{i} \bmod$ $P \neq 0$ allows this error to be detected when $P$ is prime and greater than all digits.

To detect all identical transcription errors at multiple locations, the condition in column 3 must hold for the sum of the weights at those locations. Thus, to detect all possible twin errors, the system must require that at every adjacent pair of positions the size of the error times the sum of the weights at those positions is not a multiple of $P$. Corresponding conditions, shown in columns 4 and 5, are required when $P$ is larger than all of the digits or when it is both larger and prime. The conditions for detecting jump twin errors are similar, except that the sum is taken of weights at positions that are one apart rather than adjacent. To detect multiple identical transcription errors at any combination of positions, the weights and $P$ must be chosen so that the following condition holds: $\left[\left(w_{i}+\ldots+w_{k}+\ldots+w_{m}\right)\right.$ $\times S] \bmod P \neq 0$, where the sum represents the sum of any combination of the weights. Similar conditions are required when $P$ has the restrictions described above. As discussed above, except for errors involving the first and last digit, this condition holds for the breeding program example which allows this system to detect all identical transcription errors at any combination of positions. Briggs notes that this condition will hold in any system in which $P$ $=97, w_{i}=i$, and $n<14$, and in one in which $P$ $=11, n<5$, and weight $w_{i}=i$, where $n$ is the number of digits in the identification number (Briggs, 1971).

For transcription errors, the detection condition given above can be found by subtracting the checksum equations of the erroneous and correct numbers. Similarly, according to Gallian (1996), if Eq. 1 holds, then a transposition error at positions $i$ and $j$ will not be detected if and only if $\left[\left(w_{i}-w_{j}\right) \times\left(d_{i}-d_{j}\right)\right] \bmod P=0$. Therefore a system can detect a transposition

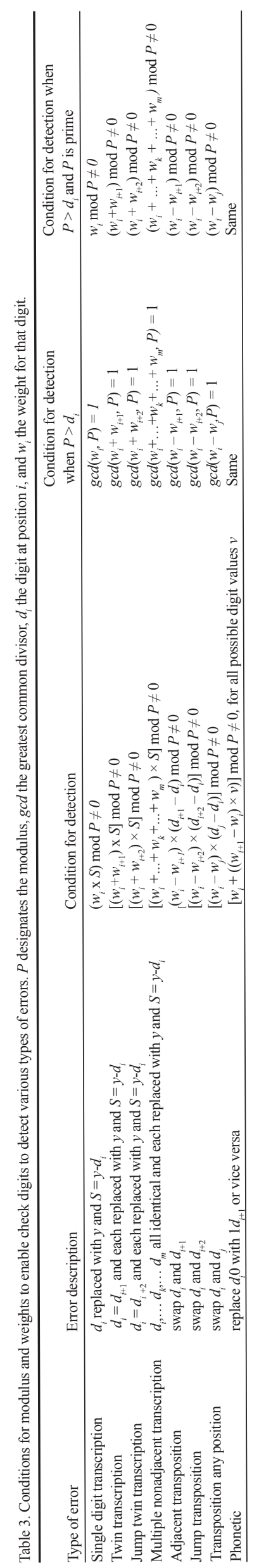


error in a pair of digits in positions $i$ and $j$ if the condition $\left[\left(w_{i}-w_{j}\right) \times\left(d_{i}-d_{j}\right)\right] \bmod P \neq 0$ holds. Conditions for detecting variations of the transposition errors given various assumptions on the value of $P$ are shown in Table 3. For a system to detect the final category of error, a phonetic error at positions $i$ and $i+1$, it must meet the following condition: $\left[\left(w_{i} \times v\right)+\left(w_{i+1}\right.\right.$ $\times 0)]-\left[\left(w_{i} \times 1\right)+\left(w_{i+1} \times v\right)\right] \bmod P \neq 0$, for all possible digit values $v$.

An equivalent but simplified form of this condition is shown in Table 3. The simplified form is used in all three columns since the restrictions on $P$ do not provide any further simplification of the condition.

Having examined the conditions needed to detect the various errors in Table 3 , it is useful to revisit some of the factors involved in the choice of $P$. Better error checking occurs when $P$ is greater than all possible values of the digits of the number. However, there is a tradeoff to be made if the difference between $P$ and the largest possible digit is more than one, because in such systems, additional symbols are needed to represent the additional check digit values. For a decimal system, a modulus of 10 would avoid the need for additional symbols. However, Gumm (1985) has shown that if a system has an even value for $P$, and if the system detects all single digit errors, then it will not detect at least one transposition error at each pair of positions. Thus for a decimal system to detect all single digit errors and all transposition errors the modulus must be 11 or larger and at least one additional character must be used for representing the check digit. Another condition that improves error checking is for $P$ to be a prime number. When this is the case, the condition required for detecting a given error is easier to meet with a set of weights, and this either makes it easier to select a set of weights that can detect a given set of errors or it makes a given set of weights more likely to detect a given set of errors.

For a given set of identification numbers, larger values of $P$ in general provide better error checking. This improvement occurs because if the set of identification numbers is partitioned into subsets that all have the same check digit, larger values of $P$ will result in a greater number of smaller subsets. If there is a need to attempt to determine the original number that resulted in the number with an error, then smaller subsets can make this easier. An example given in the last section illustrates the process of discovering the possible set of original numbers that could have produced a given erroneous number.

Common values for $P$ include 2, 7, 9, 10, $11,23,43$, and 103, and examples of systems with some of these values are given in the above section. Closely related to the choice of $P$ in the design of an error correction system is the choice of weights. The first group of systems above uses weights that repeat and the remaining systems use weights that are not repeating. Among the former are systems that repeat groups of $1,2,3$, or 4 values. In the example systems, the weight of the check digit follows the repeating pattern for some of the systems, but for other systems the check digit has a value that does not follow the pattern set by the other weights.

\section{Error Correction}

When an error is detected it is natural to try to find out what the original identification number was and thus correct the error While this can never be done with certainty, it is possible to find out which words have the highest probability of being the original number. In the breeding program example, the check digit has 23 possible values, and so when an error occurs, it is possible to create a list of the potential correct numbers that could have been the source of the word that has the error. This list will contain about $1 / 23$ of the total number of identification numbers. For example since 99-1324 should have a check digit of h, an erroneous number such as 99 $1324 \mathrm{j}$ could have originally been one of the $1 / 23$ of the identification numbers that has $j$ as a check digit (e.g., 99-1234j) or it could have originally been $99-1324 \mathrm{~h}$.

To reduce the size of the list of potentially correct numbers the checksum (i.e., the left side of Eq. 1) can be used. For erroneous word 99-1324 $\mathrm{j}$ the checksum is calculated as: [ $(1 \times$ $9)+(2 \times 9)+(3 \times 1)+(4 \times 3)+(5 \times 2)+(6$ $\times 4)+(-1 \times 8)] \bmod 23=22$.

From this value it is possible to discover which identification numbers could have produced this erroneous word from a single transcription error. For a checksum of 22 to result only from an error at position $i$, the value of $w \times S \bmod 23$, where $S$ is the size of the error, would have to be 22 . A quick check of the weights and digits shows that this is only possible for the following pairs of weight and error size: $(1,-1),(3,-8),(4,-6),(5,9),(6$, $-4)$, and $(-1,1)$. (Remember that $-1 \bmod 23$ $=22$.) However, the errors at positions 1 and 5 could not have occurred given the values of the digits of the erroneous word at those positions, and consequently the only possible words that could have been the source of the erroneous word with a single transcription error are the four valid words $99-9324 \mathrm{j}, 99-1924 \mathrm{j}$, 99-1328j, and 99-1324h.

A similar analysis can be used to discover possible transposition errors that could have produced the invalid word. Transposition errors involving the check digit could not have produced the word $99-1324 \mathrm{j}$ and are not considered further. Considering possible errors in other positions shows that a word whose checksum is 22 could have been produced only by transpositions between a pair of digits whose positions and values in the erroneous word have the following possible relationships: 1) weights that differ by one and digit values that differ by one; 2) weights that differ by three and digit values that differ by eight; 3 ) weights that differ by four and digit values that differ by six; and 4) weights that differ by five and digit values that differ by negative nine

Given the number $99-1324$, only the first transpositions could have created this word, and this could have only occurred in positions 4 and 5 between the digits 3 and 2 . Thus, the only identification number that could have produced this word from a transposition is $99-1234 j$.

Based on the assumption that transpositions and single transcription errors are the most common errors, the most likely original identification number can be narrowed down to these five. This type of analysis is obviously quite useful; although tedious and error prone, it is easily automated. In principle there is no way of knowing which of these five values was the original; in practice there may be additional information that would make it possible to select from among them.

One type of error that can always be corrected in the breeding program system is that if a digit at a known position is lost, then the value of the digit can be recovered. Given, for example, the word 9?-1234j and treating the unknown value as a 0 , the word $90-1234 \mathrm{j}$ can be used to calculate a checksum value of 5 . To bring the checksum back to 0 , it is necessary to replace the value in position 2 with a value that will make the checksum smaller by 5 which, when doing arithmetic using modulus 23 , is the same as making it larger by 18 . Since the weight of this position is 2 , then a value of 9 in this position will make the desired change and the original word must have been 99-1234j.

As seen in the examples above, the breeding program system provides partial error correction, but in general it cannot identify the original word when an error in an unknown position occurs. This system has limited error correction ability because the value of its Hamming distance is two, which is too small for the system to provide error correction. A system's Hamming distance is defined to be the minimal distance between any pair of valid words in the system. In this context, the distance between any two words is defined to be the number of digits that must be changed to convert one of the words into the other. It is not difficult to see that the Hamming distance of the breeding program system is two: changing any single digit of a valid word makes the word invalid, and so there are no pairs whose distance is one. However, changing one data digit and making a corresponding change to the check digit will create another valid word, and so there are many pairs whose distance is two. Consequently the minimal distance between any two pairs is two. In general, when the Hamming distance of a system is $d$, we say that it is a distance $d$ system.

A system with Hamming distance $d$ can detect errors in $d-1$ or fewer digits since such errors always produce a word that is not valid. Alternatively, a distance $d$ system can be used to correct errors in floor $[(d-1) / 2]$ digits, where floor $(x)$ is the largest integer less than or equal to $x$. By this criterion, the breeding program system can detect errors in one digit but it cannot be used for full error correction.

In contrast, a system with a Hamming distance of five, for example, could be used either to detect errors in four digits or to correct errors in two digits, but not both. That is, if this system is used for detection only, it is guaranteed to detect the presence of up to four errors and may detect the presence of more than 
four errors. If it is used for correction, then it will do valid correction when there are one or two errors, but if there are three or four errors then the correction process is not guaranteed to identify the actual original word. More precisely, when four or fewer errors occur it is guaranteed to detect the errors, and when more than four occur the system may or may not detect their presence. If an error is detected then the invalid word will be corrected to a word that is presumed to be the original. If there were two or fewer errors, then corrected word was actually the original. However if three or more errors occurred, then the corrected word may or may not be the actual original.

A distance five system can detect the presence of errors in up to 4 digits because when such errors occur, the word that results must be invalid. The system can correct up to 2 errors because when 1 or 2 errors occur, the invalid word that results will be closer to the original word than to any other valid word. The errors can be corrected by discovering this closest word and recognizing it as the original. However, when 3 (or 4 ) errors occur, the invalid word will be a distance of 3 (or 4) from the original word, but it may only be distance of 2 (or 1 ) from some other valid word that is not the original. In this case, the presence of the errors is detected and the closest valid word is presumed to be the original, even though it may not be.

The cause of the invalid correction is that while this system can detect the presence of up to four errors, it cannot distinguish between the case of one or two errors, when correction works, and the case of three or four errors, when correction does not work. Increasing the Hamming distance by 1 , from 5 to 6 , improves the situation by allowing the system to distinguish between the case of 3 errors and the case of 1 , 2,4 , or 5 errors. It can do this because when three errors occur, no valid word can be closer to the invalid word than the original word. Thus when this system is used for correction it provides valid correction when two errors occur, can avoid invalid correction when three errors occur, and when four or five error its correction is not guaranteed to be valid.

In general, a code of Hamming distance (2 $\times k)+1$ can be used to detect and correct errors in $k$ or fewer digits or to detect errors in $2 \times k$ or fewer digits, but it cannot be used for both simultaneously. If more than $k$ errors occur, then a valid word other than the original may be thought to be the original. However, in a code that has a Hamming distance of $(2 \times k)+$ 2 , errors in $k$ or fewer digits can be corrected, errors in $k+1$ digits will be detected, and for errors in $k+2$ or more digits an invalid correction may occur. (Hill, 1986)

A system similar to the ISBN numbers that uses four check digits can be used to create a code with distance 5 that can correct all single and double digit errors (Hill, 1986; 1990). In this code, the four check digits are chosen so that the following equations hold:

$$
\begin{aligned}
& \left(\begin{array}{l}
10 \\
\sum_{i=1} d_{i}
\end{array}\right) \bmod 11=0 \\
& \left(\begin{array}{l}
10 \\
\sum_{i=1} i \times d_{i}
\end{array}\right) \bmod 11=0 \\
& \left(\begin{array}{l}
10 \\
\sum_{i=1}^{2} \times d_{i}
\end{array}\right) \bmod 11=0 \\
& \left(\begin{array}{l}
10 \\
\sum_{i=1}^{3} \times d_{i}
\end{array}\right) \bmod 11=0
\end{aligned}
$$

Using just 2 check digits and the first 2 equations gives a distance three system that can correct all single digit errors. This basic technique can be extended to correct any number of errors as long as it is possible to increase the size of the word.

\section{Conclusion}

Check digits for error detection are widely used in a variety of applications to detect commonly occurring errors, and they could be applied to any research or commercial setting where many numbers are used. A potential user should study their particular needs relative to the types of numbers used and the most likely types of errors that occur. These parameters influence the choice of modulus and weights employed. Check codes can then be calculated in spreadsheets or in a program such as SAS (StatisticalAnalysis System). Where bar codes are conveniently used, they can incorporate the detection codes.

\section{Literature Cited}

Beckley, D.F. 1967. An optimum system with 'modulus 11'. Computer Bul. 11:309-311.

Briggs, T. 1971. Weights for Modulus 97 systems. Computer Bul. 15:79.

Gallian, J. 1996. Error detection methods. ACM Computing Surveys 28:504-517.

Gumm, H.P 1985. Anew class of check digit methods for arbitrary number systems. IEEE Trans. Info. Theory 31:102-105.

Hamming, R.W. 1980. Coding and information theory. Prentice Hall, Englewood Cliffs, N.J.

Hill, R. 1986. A first course in coding theory. Oxford Univ. Press, New York.

Hill, R. 1989. Error correcting codes I. Math. Spectrum 22:94-102.

Hill, R. 1990. Error correcting codes II. Math. Spectrum 23:14-22.

Kirtland, J. 2001. Identification numbers and check digit schemes. Math. Assn. Amer., Wash, D.C.

Knuth, D.E. 1997. The art of computer programming vol. 1. Fundamental algorithms. 3rd ed. Addison Wesley, Reading, Mass.

Verhoeff, J. 1969. Error detecting decimal codes. Mathematical Centre, Amsterdam.

Wild, W.G. 1968. The theory of Modulus N check digit systems. Computer Bul. 12:309-311. 